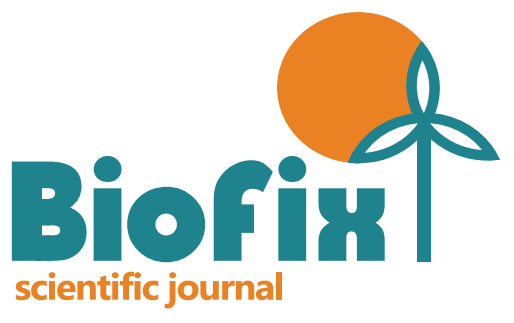

v. 2 n. 12017

Recebido em 19/02/2017

Aceito em 11/05/2017

Publicado em 15/05/2017

DOI: dx.doi.org/10.5380/biofix.v2i1.50761

\section{COMPARAÇÃO DE MÉTODOS E PROCESSOS DE AMOSTRAGEM PARA INVENTÁRIO EM FLORESTA OMBRÓFILA MISTA}

\author{
COMPARISON OF METHODS AND SAMPLING PROCEDURES FOR \\ INVENTORY IN ARAUCARIA FOREST
}

\author{
Julia Diogo Sydow ${ }^{1}$ \\ Carlos Roberto Sanquetta ${ }^{2}$ \\ Ana Paula Dalla Corte ${ }^{3}$
}

Mateus Niroh Inoue Sanquetta ${ }^{4}$

Afonso Figueiredo Filho ${ }^{5}$

\begin{abstract}
Universidade Federal do Paraná, Curitiba, Paraná, Brasil juliadsydow@gmail.com ${ }^{1}$,carlos_sanquetta@hotmail.com ${ }^{2}$, anapaulacorte@gmail.com ${ }^{3}$ \& mateus.sanquetta@hotmail.com ${ }^{4}$
\end{abstract}

Universidade Estadual do Centro-Oeste, Irati, Paraná, Brasil afonso@irati.unicentro.br

\section{RESUMO}

Inventários florestais são indispensáveis para avaliar quantitativa e qualitativamente os recursos existentes em determinada propriedade ou região. Diferentes métodos e processos aplicados aos inventários repercutem em estimativas com maior ou menor precisão. O objetivo deste estudo foi comparar as dimensões de unidades amostrais, sua distribuição na população e intensidades amostrais na estimativa da densidade, área basal e volume por hectare em um fragmento de Floresta Ombrófila Mista no Sul do estado do Paraná. Foram analisadas 28 configurações de amostragem, as quais foram avaliadas por meio dos respectivos erros de amostragem relativos em uma área submetida ao censo e comparadas a partir do erro real relativo. Foram testadas duas frações de amostragem, correspondendo a 8 e $16 \%$ da área da população, considerando os processos aleatório e sistemático. Foram avaliados oito tamanhos de unidade amostrais de 400 a $1.050 \mathrm{~m}^{2}$. As configurações amostrais avaliadas resultaram em erros relativos inferiores a $20 \%$ para as três variáveis, valores esses considerados aceitáveis para florestas nativas. Os menores erros reais relativos foram encontrados para unidades amostrais de $1.000 \mathrm{~m}^{2}$ para as três variáveis estudadas, variando apenas na sua forma, sendo $25 \mathrm{~m} \times 40 \mathrm{~m}$ para o número de árvores por hectare e $10 \mathrm{~m} \times 100$ $\mathrm{m}$ para área basal e volume por hectare. Concluiu-se que maior fração amostral gera estimativas mais precisas para número de árvores por hectare e área basal. A forma das unidades amostrais influenciou os resultados.

PALAVRAS-CHAVE: Amostragem, Araucária, Censo florestal.

\section{ABSTRACT}

Forest inventories are essential to evaluate quantitatively and qualitatively the existing resources in a given property or region. Different methods and processes applied to inventories provide estimates with more or less precision level. The aim of this study was to compare different sizes of sample units, their sampling design, and sampling intensity to estimate tree density, basal area and volume per hectare in a remnant of Araucaria Forest in southern Parana State. We tested 28 sample design, which were evaluated by the respective sampling errors in an area under census. and compared by the relative real error. We compared two sampling areas, corresponding to 6 and $16 \%$ of sampling fraction, considering the random and systematic sampling designs. Eight sample unit sizes were evaluated, ranging from 400 to $1.050 \mathrm{~m}^{2}$. All the tested sample treatments resulted in a relative error less than $20 \%$ for the three variables analyzed, which is satisfactory for native forests. The lowest real relative errors were found for sample units of 1.000 $\mathrm{m}^{2}$ for the three variables, varying only in their form, being $25 \mathrm{~m} \mathrm{x}$ $40 \mathrm{~m}$ for tree density and $10 \mathrm{~m} \times 100 \mathrm{~m}$ for basal area and volume per hectare. The larger the sample area the more accurate were the estimates. It was concluded that larger sampling fraction results more accurate estimates for tree density and basal area. The sampling design influence the estimates.

KEYWORDS: Sampling, Araucaria, Forest census. 


\section{INTRODUÇÃO}

Inventários florestais se constituem em uma atividade de grande importância sob vários aspectos, sendo imprescindível para o conhecimento do potencial dos recursos madeireiros e não madeireiros existentes em determinada área. No estabelecimento, manutenção ou ampliação da produção das indústrias que utilizam matéria-prima florestal, deve-se ter o conhecimento da disponibilidade quantitativa e qualitativa dos recursos florestais. Isso é possível com a realização de inventários florestais (SANQUETTA et al., 2014).

Em florestas de grandes dimensões, a mensuração da população a $100 \%$, conhecida como censitária, é uma atividade impraticável devido ao custo e ao tempo. Dessa forma, se faz necessária a aplicação de métodos e processos de amostragem adequados na realização do inventário florestal, visando à obtenção de informações quantitativas e qualitativas confiáveis sobre os recursos existentes. O êxito na obtenção de informações precisas oriundas de um inventário florestal está intimamente relacionado aos processos e métodos de amostragem a serem adotados, assim como à intensidade amostral adotada (UBIALLI et al., 2009; CORTE et al., 2013).

$\mathrm{O}$ tamanho das unidades amostrais é um fator crucial na execução de um inventário florestal. O tamanho e a forma das unidades amostrais exercem influência muito importante sobre a precisão das estimativas (SINGH, 1974). Em geral, os coeficientes de variação decrescem inversamente ao tamanho das unidades amostrais, e, consequentemente, quanto menor for a unidade de amostra, maior quantidade de unidades será necessária para obter o mesmo grau de precisão (MORAIS FILHO et al., 2003).

Em florestas nativas, geralmente, observa-se grande variabilidade dimensional das unidades amostrais adotadas, o que pode repercutir em grande número de valores zero se forem utilizadas unidades amostrais pequenas, afetando a distribuição normal dos elementos na floresta (SCOLFORO; MELLO, 2006). Por outro lado, quando se trata de florestas relativamente homogêneas, a precisão tende a ser maior para unidades amostrais pequenas em relação às maiores, uma vez que o número de unidades amostrais independentes é grande (HUSCH et al., 1982; BONETES, 2003).

A configuração das unidades amostrais na população, o que caracteriza o processo de amostragem em inventários de comunidades vegetais, deve ser estabelecida em função do objetivo do levantamento e das variáveis mais importantes (DURIGAN, 2009). Os processos mais empregados, de maneira geral, são o aleatório, o sistemático e o misto, simples ou estratificados, que devem ser eleitos de acordo com as características da população (SANQUETTA et al., 2014).

Embora existam estudos sobre processos e métodos de amostragem em inventários de florestas nativas (BONETES, 2003; UBIALLI et al., 2009; AUGUSTYNCZIK, 2011), são raras as pesquisas que relacionam os três elementos fundamentais de um sistema de inventário florestal: i) área amostrada; ii) processo de amostragem; e iii) tamanho da unidade de amostra, considerando a comparação direta com o censo florestal.

Este estudo objetivou comparar o desempenho de diferentes tamanhos de unidades de amostra (método), seus desenhos de distribuição (processo) e intensidade amostral, a fim de indicar as combinações adequadas para estimação de número de árvores, área basal e volume por hectare em um fragmento de Floresta Ombrófila Mista do Sul do estado do Paraná.

\section{MATERIAL E MÉTODOS}

\section{Local de estudo e dados empregados}

Este trabalho foi realizado na FLONA (Floresta Nacional) de Irati, localizada no munícipio de Fernandes Pinheiro, zona fisiográfica de Irati, situado no Planalto Paranaense, ao Sul do estado do Paraná (Figura 1). A área tem como referência geográfica a interseção do paralelo $25^{\circ} 27^{\prime} 56^{\prime \prime}$ latitude Sul e o meridiano $50^{\circ} 37^{\prime} 51^{\prime \prime}$ de longitude Oeste. A altitude média do local é de $820 \mathrm{~m}$ acima do nível médio do mar, apresentando relevo suavemente ondulado. De acordo com a classificação de Köppen, o clima da região é do tipo Cfb, Subtropical Úmido Mesotérmico, com verões amenos, ocorrência de geadas severas e frequentes e ausência de estação seca (MAZZA et al., 2005).

A área de estudo é uma Unidade de Conservação de Uso Sustentável vinculada ao Instituto Chico Mendes de Conservação da Biodiversidade (ICMBio), apresentando características favoráveis para o desenvolvimento de pesquisas científicas sobre o ecossistema Floresta Ombrófila Mista (FOM) ou Floresta com Araucária. A FLONA de Irati possui superfície total de 3.495 ha, sendo $57,6 \%$ ocupados por florestas nativas com predominância de araucária (Araucaria angustifolia (Bert.) Kuntze) e espécies folhosas associadas a diferentes famílias, tais como canelas e imbuias (Lauraceae) e angicos (Fabaceae). O restante da área é ocupado por plantações florestais de A. angustifolia, Pinus elliottii Engelm. e Pinus taeda L., dentre outras (FIGUREIDO FILHO, 2010). 

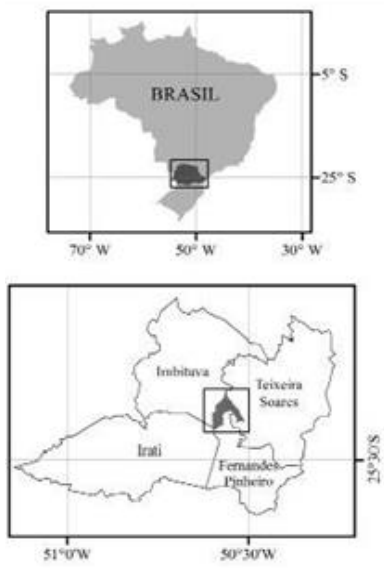
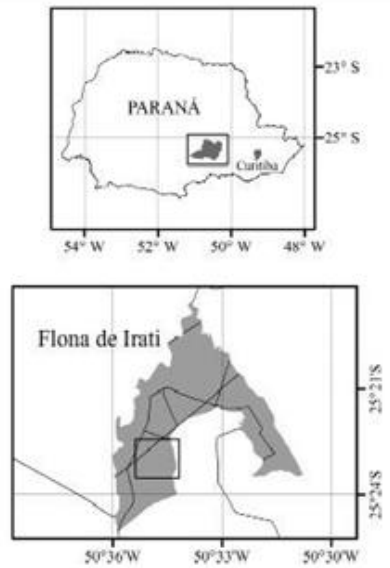
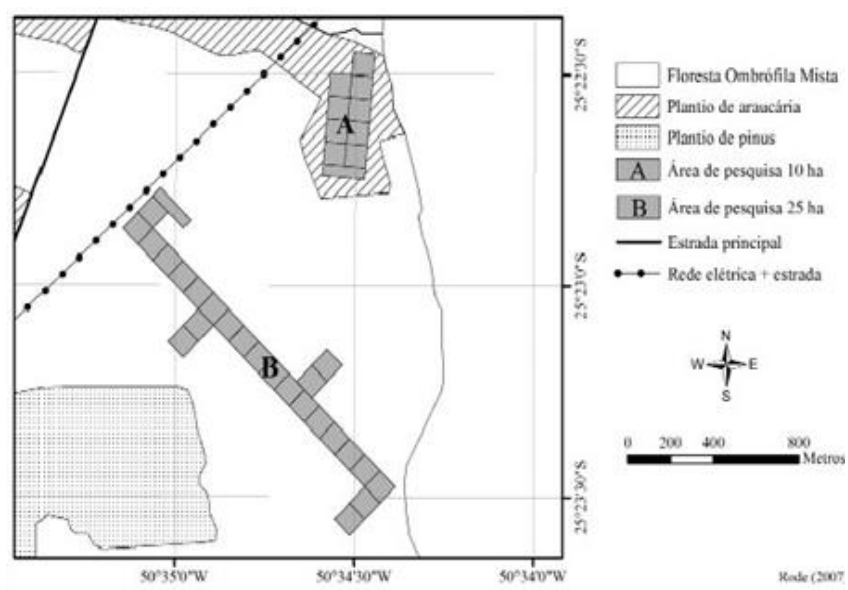

Figura 1. Localização da área de estudo e da FLONA de Irati (Fonte: RODE, 2008).

No ano de 2002 foi implantado um conjunto de unidades amostrais permanentes num fragmento de FOM situado na FLONA, com o propósito de estudar sua dinâmica de crescimento e regeneração. O estudo foi idealizado pelo Laboratório de Manejo Florestal do Curso de Engenharia Florestal da Universidade Estadual do Centro-Oeste (UNICENTRO). Nesse estudo, foram estabelecidas 25 unidades amostrais permanentes, com dimensões de $100 \mathrm{~m} \times 100 \mathrm{~m}$, totalizando 25 ha. Cada unidade amostral foi dividida em quatro subparcelas de $0,25$ ha ( $50 \mathrm{~m} \times 50 \mathrm{~m})$, com controle em faixas de $10 \mathrm{~m} \times 50$ $\mathrm{m}(0,05 \mathrm{ha})$ (CORTE et al., 2013). Todas as árvores com dap (diâmetro à $1,30 \mathrm{~m}$ do solo) superior a $10 \mathrm{~cm}$ foram incluídas e mapeadas em um sistema cartesiano $X Y$ (PÉLLICO NETTO et al., 2012). Remedições foram realizadas também nos anos de 2005 e 2008.

Em 2008, a remedição das 25 unidades amostrais permanentes foi feita em $100 \%$ dos indivíduos arbóreos. Os dados dessa remedição censitária foram utilizados no atual trabalho como o parâmetro da população. Maiores detalhes podem ser encontrados em ROIK (2012) e em PÉLLICO NETTO et al, 2012).

\section{Configuração dos procedimentos de amostragem}

Neste estudo, foi aplicada a metodologia proposta por Corte et al. (2013), considerando diferentes configurações amostrais para fins de comparação, conforme descrito a seguir. Assim, três fatores experimentais foram examinados:

\section{Fator 1 - Fração de amostragem}

Foram definidas duas áreas totais amostradas, de 2 e 4 ha, correspondentes às frações de amostragem de 8 e 16\%, respectivamente. Para cada fração de amostragem, calculou-se o número de unidades de amostras necessárias em função do tamanho das mesmas e das respectivas intensidades amostrais. A escolha por esses percentuais baseou-se no trabalho publicado por Magurran (2011) e Corte et al. (2013). Adicionalmente, pode-se dizer que estão condizentes com a magnitude e amplitude adotada por Augustynczik (2011).

Fator 2 - Tamanho das unidades amostrais

Foram selecionadas sete dimensões distintas de unidades amostrais, com variação de 500 a $1.000 \mathrm{~m}^{2}$, totalizando 14 combinações (Tabela 1 ). O censo florestal (medição a 100\%) foi considerado como a testemunha (configuração de amostragem 1).

Tabela 1. Configurações de amostragem, dimensões, áreas e número de unidades amostrais (UAs) em um fragmento de FOM na FLONA de Irati, Sul do Paraná

\begin{tabular}{|c|c|c|c|c|}
\hline & \multirow{3}{*}{ Dimensão das UAs } & \multirow{3}{*}{ Área $\left(\mathrm{m}^{2}\right)$} & \multicolumn{2}{|c|}{ Número de UAs } \\
\hline & & & \multicolumn{2}{|c|}{ Fração de amostragem } \\
\hline & & & $8 \%$ (2 ha) & $16 \%$ (4 ha) \\
\hline 1 & \multicolumn{4}{|c|}{ Censo Florestal } \\
\hline 2 & $10 \mathrm{~m} \times 50 \mathrm{~m}$ & 500 & 40 & 80 \\
\hline 3 & $10 \mathrm{~m} \times 100 \mathrm{~m}$ & 1.000 & 20 & 40 \\
\hline 4 & $20 \mathrm{~m} \times 20 \mathrm{~m}$ & 400 & 50 & 100 \\
\hline 5 & $20 \mathrm{~m} \times 25 \mathrm{~m}$ & 500 & 40 & 80 \\
\hline 6 & $20 \mathrm{~m} \times 50 \mathrm{~m}$ & 1.000 & 20 & 40 \\
\hline 7 & $30 \mathrm{~m} \times 35 \mathrm{~m}$ & 1.050 & 19 & 38 \\
\hline 8 & $25 \mathrm{~m} \times 40 \mathrm{~m}$ & 1.000 & 20 & 40 \\
\hline
\end{tabular}

Fator 3 - Processo de amostragem

Foram analisados dois processos de amostragem: aleatório e sistemático. Dessa maneira, o estudo testou 28 configurações de amostragem para fins de comparação (Figura 2). 


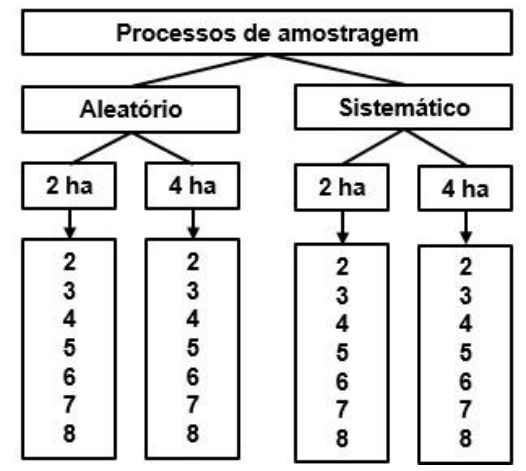

Figura 2. Combinações de amostragem testadas para inventário florestal de fragmento de FOM na FLONA de Irati, Sul do Paraná.

\section{Variáveis analisadas}

Três variáveis da população florestal foram analisadas: número de árvores, área basal e volume total, todas para a unidade de um hectare. Com base nessas variáveis, aplicou-se a análise estatística das estimativas, com o cálculo dos respectivos erros de amostragem relativos.

O valor paramétrico do número de árvores por hectare (N) foi determinado com base no censo florestal, englobando todas as espécies. As estimativas foram geradas por meio das seguintes equações:

$$
\begin{aligned}
& N_{j}=m_{j} F \\
& N=\frac{\sum_{j=1}^{n} N_{j}}{n} \\
& F=\frac{1}{a}
\end{aligned}
$$

Em que:

$\mathrm{Nj}$ = número de árvores por j-ésima unidade amostral;

$N=$ número médio de árvores por hectare;

$F=$ fator de proporcionalidade;

$a=$ área de unidade amostral (ha);

$m_{j}=$ número de árvores na j-ésima unidade amostral; e $n=$ número de unidades amostrais.

O valor paramétrico da área basal por hectare foi obtido com base no censo florestal, a partir da soma das áreas transversais $\left(g_{i}\right)$ dos indivíduos arbóreos com $d a p_{i}$ acima de $10 \mathrm{~cm}$ nela inseridos.

$$
\begin{aligned}
& g_{i}=\frac{\pi \text { dap }_{i}^{2}}{40.000} \\
& G_{j}=\sum_{i=1}^{m} g_{i} F \\
& G=\frac{\sum_{j=1}^{n} G_{j}}{n}
\end{aligned}
$$

Em que:

$g_{i}=$ área transversal de cada i-ésima árvore $\left(\mathrm{m}^{2}\right)$;

$G_{j}=$ área basal por j-ésima unidade amostral $\left(\mathrm{m}^{2}\right)$;

$G$ = área basal média por hectare $\left(\mathrm{m}^{2} \mathrm{ha}^{-1}\right)$; e

$\operatorname{dap}_{i}=$ diâmetro a 1,3 $\mathrm{m}$ do solo de cada i-ésima árvore na j-ésima unidade amostral $(\mathrm{cm})$.

O valor paramétrico da variável volume com casca por hectare foi calculado a partir do censo florestal. Como as alturas das árvores contidas na população não foram medidas em 2008, seus valores foram estimados a partir das seguintes equações (PIZATTO, 1999):

Para a araucária:

$$
h_{i}=\frac{-56,243247+17,575912 \text { dap }_{i}+0,112721 \text { dap }_{i}^{2}}{\operatorname{dap}_{i}}
$$

$R^{2}{ }_{a j .}=0,70, S y x \%=14,17 \%, n=110$

Para as demais espécies:

$$
h_{i}=\frac{-79,852966+20,005797 d_{d a p_{i}}+0,113487 \text { dap }_{i}{ }^{2}}{\operatorname{dap}_{i}}
$$

$R^{2}{ }_{a j .}=0,64$, Syx\% $=16,21 \%, n=97$

Em que:

$h_{i}=$ altura total individual da i-ésima árvore $(\mathrm{m})$;

Para o cálculo do volume individual de cada i-ésima árvore, empregou-se a seguinte equação (VIBRANS et al., 2013):

$$
\ln \left(\frac{v_{i}}{1.000}\right)=-17,96+0,96 \ln \left(\operatorname{cap}_{i}\right)+\ln \left(h_{i}\right)
$$

$R^{2}{ }_{a j .}=0,94, n=606$

Em que:

$v_{i}=$ volume total do fuste da i-ésima árvore com casca $\left(\mathrm{m}^{3}\right)$; $c a p_{i}=$ circunferência à 1,3 $\mathrm{m}$ do solo da i-ésima árvore na jésima unidade amostral $(\mathrm{cm})$; e In = logaritmo neperiano.

A partir do volume de cada i-ésima árvore, foi calculado o volume de cada j-ésima unidade amostral e a média por hectare, como segue:

$$
\begin{gathered}
V_{j}=\sum_{i=1}^{m} v_{i} F \\
V=\frac{\sum_{j=1}^{n} v_{j}}{n}
\end{gathered}
$$

Em que:

$V_{j}=$ volume por unidade amostral; e

$V=$ volume médio por hectare. 


\section{Erro de amostragem}

A utilização de processos de amostragem implica em erros de amostragem, devido a medição de apenas parte da população. Nesse sentido, Bonetes (2003) afirma que quanto menor o erro de amostragem, mais precisas serão as estimativas e extrapolações realizadas. Assim, o erro de amostragem indica o quão próximo o valor estimado está do correspondente valor verdadeiro obtido pelo censo. Foram calculados os erros de amostragem relativos para as três variáveis estudadas, de acordo com a seguinte equação (SANQUETTA et al., 2014):

$$
\begin{aligned}
& E A(\%)= \pm \frac{E_{a}}{\bar{x}} \cdot 100 \\
& E_{a}= \pm t s_{\bar{x}}
\end{aligned}
$$

Em que:

$E_{a}=$ erro de amostragem absoluto;

$t=$ valor tabelar de Student para 95\% de probabilidade; e

$S_{\bar{x}}=$ erro padrão ou desvio padrão da média

Faz-se necessário ressaltar que, fundamentalmente, a amostragem sistemática não produz estimativas do erro de amostragem válidas, pois são exigidas pelo menos duas unidades amostrais distribuídas aleatoriamente (BONETES, 2003). Nesse sentido, Péllico Netto e Brena (1997) afirmam que a alocação da primeira unidade amostral de forma aleatória, sendo as demais equidistantes, permite o cálculo do erro de amostragem como uma amostra aleatória.

\section{Critério de comparação entre as configurações de amostragem}

Após o cálculo dos parâmetros obtidos com base no censo, das estimativas através de amostragem e dos seus respectivos erros relativos, foi determinada a diferença entre o valor paramétrico e o valor da estimativa. Essa comparação direta possibilitou diagnosticar o erro real que se obtém ao realizar a amostragem de uma parte da população.

$$
E R=\frac{(V E-V R)}{V R} .100
$$

Em que:

$E R=$ Erro real relativo (\%);

$V E=$ Valor estimado pela amostragem; e

$V R=$ Valor real resultante do censo.

Com base nos estudos realizados por Ubialli (2007) e
Augustynczik (2011), o erro real foi utilizado para comparar os valores paramétricos obtidos através do censo, com as estimativas obtidas nas simulações de amostragem.

\section{RESULTADOS E DISCUSSÃO}

\section{Parâmetros da população}

Os valores paramétricos de $N, G$ e $V$ por hectare foram 571 árvores, $29,7870 \mathrm{~m}^{2}$ e $342,1000 \mathrm{~m}^{3}$, respectivamente. Esses valores são compatíveis com outras florestas em condições semelhantes, como Corte et al. (2014), embora existam diferenças caso a caso, dependendo do estágio de regeneração, da composição e da estrutura da floresta.

\section{Variáveis analisadas}

Número de árvores por hectare

Os erros relativos de amostragem variaram de 5,45\% (unidade amostral de $20 \mathrm{~m} \times 20 \mathrm{~m}$, fração de amostragem $16 \%$, amostragem sistemática) a 14,68\% (unidade amostral de $25 \mathrm{~m} \times 40 \mathrm{~m}$, fração de amostragem 8\%, amostragem sistemática) para o número de árvores por hectare.

A melhor configuração de amostragem subestimou o número de árvores pode hectare em $0,18 \%$ (unidade amostral de $25 \mathrm{~m} \times 40 \mathrm{~m}$, fração de amostragem 16\%, amostragem aleatória) (Tabela 2).

Área basal por hectare

Para a área basal, a variação foi de 5,65\% (unidade amostral de $20 \mathrm{~m} \times 25 \mathrm{~m}$, fração de amostragem 16\%, amostragem sistemática) a 13,82\% (unidade amostral de $20 \mathrm{~m} \times 50 \mathrm{~m}$, fração de amostragem 8\%, amostragem aleatória).

A configuração de amostragem que obteve a melhor estimativa para a variável área basal por hectare subestimou o valor paramétrico em $0,20 \%$ (unidade amostral de $10 \mathrm{~m} \times 100$ m, fração de amostragem 16\%, amostragem sistemática).

Volume por hectare

Para o volume, os erros relativos variaram de $6,26 \%$ (unidade amostral de $20 \mathrm{~m} \times 25 \mathrm{~m}$, fração de amostragem $16 \%$, amostragem sistemática) a 15,96\% (unidade amostral de 25 m x 40 m, fração de amostragem 8\%, amostragem aleatória). 
Tabela 2. Estimativas do número de árvores em um remanescente de FOM na FLONA de Irati, Sul do Paraná, e respectivos erros de amostragem relativos em diferentes procedimentos de amostragem

\begin{tabular}{|c|c|c|c|c|c|c|c|c|c|c|c|c|}
\hline \multirow{3}{*}{$\begin{array}{l}\text { Configuração } \\
\text { de amostragem }\end{array}$} & N/ha & $E A(\%)$ & $E R(\%)$ & N/ha & $E A(\%)$ & $E R(\%)$ & N/ha & $E A(\%)$ & $E R(\%)$ & N/ha & $E A(\%)$ & $E R(\%)$ \\
\hline & \multicolumn{6}{|c|}{ Amostragem Aleatória } & \multicolumn{6}{|c|}{ Amostragem Sistemática } \\
\hline & \multicolumn{3}{|c|}{$8 \%$} & \multicolumn{3}{|c|}{$16 \%$} & \multicolumn{3}{|c|}{$8 \%$} & \multicolumn{3}{|c|}{$16 \%$} \\
\hline 1 (Censo) & \multicolumn{3}{|c|}{571} & \multicolumn{3}{|c|}{571} & \multicolumn{3}{|c|}{571} & \multicolumn{3}{|c|}{571} \\
\hline $2(10 \mathrm{~m} \times 50 \mathrm{~m})$ & 554 & 9,75 & $-3,06$ & 580 & 7,01 & 1,53 & 595 & 7,86 & 4,12 & 580 & 6,48 & 1,93 \\
\hline $3(10 \mathrm{~m} \times 100 \mathrm{~m})$ & 539 & 12,47 & $-5,60$ & 551 & 6,86 & $-3,59$ & 578 & 12,69 & 1,14 & 551 & 6,17 & $-1,49$ \\
\hline $4(20 \mathrm{~m} \times 20 \mathrm{~m})$ & 547 & 9,32 & $-4,29$ & 588 & 6,59 & 2,98 & 580 & 9,27 & 1,49 & 588 & 5,45 & 2,58 \\
\hline $5(20 \mathrm{~m} \times 25 \mathrm{~m})$ & 561 & 10,28 & $-1,84$ & 536 & 7,63 & $-6,09$ & 529 & 8,35 & $-7,44$ & 536 & 6,37 & 7,27 \\
\hline $6(20 \mathrm{~m} \times 50 \mathrm{~m})$ & 604 & 13,75 & 5,78 & 555 & 11,65 & $-2,89$ & 564 & 11,76 & $-1,31$ & 555 & 6,76 & 0,57 \\
\hline $7(30 \mathrm{~m} \times 35 \mathrm{~m})$ & 574 & 12,01 & 0,51 & 569 & 7,46 & $-0,41$ & 551 & 12,34 & $-3,44$ & 569 & 7,17 & $-3,35$ \\
\hline $8(25 \mathrm{~m} \times 40 \mathrm{~m})$ & 534 & 11,54 & $-6,57$ & 570 & 10,00 & $-0,18$ & 582 & 14,68 & 1,93 & 570 & 7,15 & $-0,31$ \\
\hline
\end{tabular}

Tabela 3. Estimativas da área basal em um remanescente de FOM na FLONA de Irati, Sul do Paraná, e respectivos erros de amostragem relativos em diferentes procedimentos de amostragem

\begin{tabular}{|c|c|c|c|c|c|c|c|c|c|c|c|c|}
\hline \multirow{3}{*}{$\begin{array}{l}\text { Configuração } \\
\text { de amostragem }\end{array}$} & $G\left(\mathrm{~m}^{2} h \mathrm{a}^{-1}\right)$ & $E A(\%)$ & $E R(\%)$ & N/ha & $E A(\%)$ & $E R(\%)$ & $G\left(m^{2} h^{-1}\right)$ & $E A(\%)$ & $E R(\%)$ & $N /$ ha & $E A(\%)$ & ER (\%) \\
\hline & \multicolumn{6}{|c|}{ Amostragem Aleatória } & \multicolumn{6}{|c|}{ Amostragem Sistemática } \\
\hline & \multicolumn{3}{|c|}{$8 \%$} & \multicolumn{3}{|c|}{$16 \%$} & \multicolumn{3}{|c|}{$8 \%$} & \multicolumn{3}{|c|}{$16 \%$} \\
\hline 1 (Censo) & \multicolumn{3}{|c|}{29,7870} & \multicolumn{3}{|c|}{29,7870} & \multicolumn{3}{|c|}{29,7870} & \multicolumn{3}{|c|}{29,7870} \\
\hline $2(10 \mathrm{~m} \times 50 \mathrm{~m})$ & 29,4200 & 10,70 & $-1,23$ & 30,449 & 7,41 & 2,22 & 29,0700 & 9,19 & $-2,41$ & 30,4290 & 7,34 & 2,16 \\
\hline $3(10 \mathrm{~m} \times 100 \mathrm{~m})$ & 27,5510 & 10,76 & $-7,50$ & 29,991 & 7,99 & 0,69 & 30,6740 & 11,84 & 2,98 & 29,7260 & 7,30 & $-0,20$ \\
\hline $4(20 \mathrm{~m} \times 20 \mathrm{~m})$ & 29,0150 & 11,69 & $-2,59$ & 28,900 & 6,93 & $-2,98$ & 31,7860 & 8,31 & 6,71 & 28,3220 & 5,96 & $-4,92$ \\
\hline $5(20 \mathrm{~m} \times 25 \mathrm{~m})$ & 29,1090 & 11,17 & $-2,27$ & 27,866 & 6,93 & $-6,45$ & 27,2800 & 9,95 & $-8,42$ & 30,9810 & 5,65 & 4,01 \\
\hline $6(20 \mathrm{~m} \times 50 \mathrm{~m})$ & 29,0870 & 13,82 & $-2,35$ & 28,951 & 8,62 & $-2,80$ & 29,0620 & 10,14 & $-2,43$ & 29,0310 & 6,80 & $-2,54$ \\
\hline $7(30 \mathrm{~m} \times 35 \mathrm{~m})$ & 29,5950 & 12,29 & $-0,64$ & 31,347 & 7,76 & 5,24 & 28,6550 & 9,10 & $-3,80$ & 28,6470 & 6,56 & $-3,83$ \\
\hline $8(25 \mathrm{~m} \times 40 \mathrm{~m})$ & 30,8180 & 12,88 & 3,46 & 28,769 & 8,93 & $-3,42$ & 28,9570 & 12,29 & $-2,79$ & 29,0760 & 6,24 & $-2,39$ \\
\hline
\end{tabular}

Tabela 4. Estimativas do volume em um remanescente de FOM na FLONA de Irati, Sul do Paraná, e respectivos erros de amostragem relativos em diferentes procedimentos de amostragem

\begin{tabular}{|c|c|c|c|c|c|c|c|c|c|c|c|c|}
\hline \multirow{3}{*}{$\begin{array}{l}\text { Configuração } \\
\text { de amostragem }\end{array}$} & $V\left(m^{3} h a^{-1}\right)$ & $E A(\%)$ & $E R(\%)$ & N/ha & $E A(\%)$ & $E R(\%)$ & $V\left(\mathrm{~m}^{3} h \mathrm{a}^{-1}\right)$ & $E A(\%)$ & $E R(\%)$ & N/ha & $E A(\%)$ & $E R(\%)$ \\
\hline & \multicolumn{6}{|c|}{ Amostragem Aleatória } & \multicolumn{6}{|c|}{ Amostragem Sistemática } \\
\hline & \multicolumn{3}{|c|}{$8 \%$} & \multicolumn{3}{|c|}{$16 \%$} & \multicolumn{3}{|c|}{$8 \%$} & \multicolumn{3}{|c|}{$16 \%$} \\
\hline 1 (Censo) & \multicolumn{3}{|c|}{342,1000} & \multicolumn{3}{|c|}{342,1000} & \multicolumn{3}{|c|}{342,1000} & \multicolumn{3}{|c|}{342,1000} \\
\hline $2(10 \mathrm{~m} \times 50 \mathrm{~m})$ & 332,3330 & 13,21 & $-2,85$ & 352,1040 & 8,78 & 2,92 & 332,1750 & 11,17 & $-2,90$ & 348,7850 & 7,92 & 1,95 \\
\hline $3(10 \mathrm{~m} \times 100 \mathrm{~m})$ & 303,2800 & 13,07 & $-11,35$ & 346,8370 & 9,19 & 1,38 & 342,1970 & 14,17 & 0,03 & 333,6050 & 9,01 & $-2,48$ \\
\hline $4(20 \mathrm{~m} \times 20 \mathrm{~m})$ & 324,7760 & 14,94 & $-5,06$ & 328,1590 & 8,35 & $-4,08$ & 363,9650 & 9,79 & 6,39 & 320,4710 & 7,33 & $-6,32$ \\
\hline $5(20 \mathrm{~m} \times 25 \mathrm{~m})$ & 339,5220 & 13,39 & $-0,75$ & 318,3390 & 7,99 & $-6,95$ & 311,6280 & 11,82 & $-8,91$ & 351,9830 & 6,26 & 2,89 \\
\hline $6(20 \mathrm{~m} \times 50 \mathrm{~m})$ & 318,0060 & 14,51 & $-7,04$ & 329,2120 & 10,06 & $-3,77$ & 321,7090 & 12,35 & $-5,96$ & 333,3140 & 7,76 & $-2,57$ \\
\hline $7(30 \mathrm{~m} \times 35 \mathrm{~m})$ & 341,7440 & 13,28 & $-0,10$ & 368,1110 & 9,08 & 7,60 & 331,6100 & 9,73 & $-3,07$ & 328,4800 & 7,46 & $-3,98$ \\
\hline $8(25 \mathrm{~m} \times 40 \mathrm{~m})$ & 364,4360 & 15,96 & 6,53 & 321,9500 & 10,05 & $-5,89$ & 327,1990 & 12,88 & $-4,36$ & 331,2530 & 7,19 & $-3,17$ \\
\hline
\end{tabular}

A configuração de amostragem que apresentou a melhor estimativa para a variável volume por hectare superestimou o valor paramétrico em $0,03 \%$ (unidade amostral de $10 \mathrm{~m} \times 100 \mathrm{~m}$, fração de amostragem 8\%, amostragem sistemática).

\section{Erro de amostragem}

Na maioria dos casos, os erros de amostragem foram mais baixos para a variável número de árvores, em seguida para a área basal e depois para o volume. Isso indica que a 
densidade, que advém apenas de contagem das árvores, é mais estável em comparação com a área basal, que depende dos diâmetros, bem como do volume, que depende dos diâmetros e das alturas das árvores.

Considerando que o uso dos inventários florestais, em termos gerais, é de $10 \%$ de limite de erro (SANQUETTA et al., 2014), pode-se afirmar que as estimativas geradas de $N, G$ e $V$, na maioria dos casos, atende esse limite. Porém, no caso de florestas nativas, há uma tendência de se aceitar até $20 \%$ de limite de erro, considerando a grande variabilidade normalmente encontrada.

Assim, pode-se afirmar que, de forma geral, todos as configurações de amostragem testadas neste estudo produziram estimativas satisfatórias. Observou-se, também, por meio dos resultados, que não houve nenhuma configuração de amostragem que tenha gerado estimativas com tendência ou viés, já que houve sub e superestimativas em relação ao parâmetro, mas sempre dentro do limite máximo de $20 \%$.

\section{Comparação entre as configurações de amostragem}

Constatou-se que cada variável estudada apresentou uma particularidade quanto a melhor configuração de amostragem, conforme supracitado. Considerando as três variáveis estudadas, observou-se que a fração de amostragem que apresentou as melhores estimativas para $N$ e $G$ foi a de $16 \%$, enquanto para $V$ foi de $8 \%$. Por ser o volume a variável de interesse mais importante em atividades de inventário florestal, com o objetivo de diagnosticar o potencial madeireiro, a utilização de uma menor fração de amostragem implicará na redução dos custos na execução do inventário.

$\mathrm{Na}$ aplicação da amostragem em inventários florestais, deve-se considerar simultaneamente o tamanho e a forma da unidade amostral, bem como a intensidade amostral adotada do levantamento. Há uma tendência de os especialistas em manejo florestal preferirem a adoção de unidades amostrais maiores (normalmente acima de 1.000 $\mathrm{m}^{2}$ ) em florestas nativas, devido à sua heterogeneidade (AUGUSTYNCZIK et al., 2013). Por outro lado, os especialistas em levantamentos fitossociológicos preferem as unidades amostrais de menor área (geralmente com áreas de $10 \mathrm{~m} \times 10 \mathrm{~m}, 10 \mathrm{~m} \times 20 \mathrm{~m}$ ou $20 \mathrm{~m} \times 20 \mathrm{~m}$ ).

Nesses casos, as variáveis $N$ e $G$ são de maior interesse, pois os índices fitossociológicos, como Densidade e Frequência (absolutas e relativas), são calculados a partir do número de indivíduos de uma ou de um conjunto de espécies, enquanto a Dominância (absoluta e relativa) é calculada a partir da área basal.
O tamanho e a forma das unidades amostrais são fatores de decisão pelo profissional responsável pelo planejamento e pela execução do inventário. Nesse sentido, é fundamental decidir esses quesitos adequadamente, considerando as questões estatísticas, sem se descuidar dos aspectos operacionais. Unidades amostrais muito largas em florestas nativas dificultam a sua implantação em campo, requerendo equipamentos topográficos que possam garantir que a área configurada da unidade não esteja incorreta. No caso de unidades amostrais mais estreitas e compridas, esse cuidado se torna menor, tendo em vista que basta apenas fazer o controle de inclusão ou não das árvores marginais, como no caso de $G$ e $V$.

Observou-se que as variáveis $N, G$ e $V$ foram mais bem estimadas a partir de unidades amostrais maiores, com $1.000 \mathrm{~m}^{2}$, variando apenas na sua forma, sendo $25 \mathrm{~m} \times 40$ m para $N$, e $10 \mathrm{~m} \times 100 \mathrm{~m}$ para $G$ e $V$. No tocante a $N$ e $G$, o maior tamanho das unidades amostrais influenciou na melhoria dos estimadores, confrontando com o que normalmente é empregado. Kersten e Galvão (2011), estudando um fragmento urbano de FOM no município de Curitiba - PR, notaram que a intensidade amostral repercute em maior ganho de precisão na estimativa de área basal ao tamanho da unidade amostral. Já o ganho para o caso da estimativa do número total de árvores isso se inverte.

Kersten e Galvão (2011) concluíram, também, que os melhores resultados para as estimativas de área basal e número de árvores foram alcançados com unidades amostrais de $1.000 \mathrm{~m}^{2}$. Na maior parte dos casos, percebeu-se leve superioridade do processo de amostragem sistemático em relação ao aleatório, no que concerne ao erro de amostragem relativo. Tratando-se de erros reais relativos, as estimativas das variáveis $G$ e $V$, em configurações de amostragem a partir do processo de amostragem sistemático apresentaram os valores mais satisfatórios, ou seja, os valores mais próximos de zero. No entanto, não houve como comparar a eficiência operacional, tendo em vista que as amostragens foram simuladas dentro da população submetida a censo.

O êxito da execução do inventário florestal é dependente da definição adequada do método e do processo de amostragem. A correspondente intensidade amostral é também um elemento crucial para se obter estimativas precisas. A eleição do processo de amostragem deve considerar as características da área a ser inventariada e da tipologia florestal em questão, além da distribuição espacial da variável de interesse. Em todas essas definições, deve-se considerar, ainda, a experiência 
do técnico e a disponibilidade de tempo e de recursos para sua realização (ROCHA et al., 2013).

Por fim, é importante salientar que cada população alvo de inventário florestal deve ser analisada previamente antes da realização do levantamento, planejando-se apropriadamente o método (tamanho e forma da unidade amostral), o processo de amostragem, e a intensidade de amostragem, considerando sempre o limite de erro admissível e os objetivos do usuário das informações. Não há uma recomendação válida universalmente e as especificidades da população objeto devem ser respeitadas. Levando-se em conta simultaneamente esses elementos, o profissional estará apto a decidir a melhor opção técnica para realizar um inventário florestal de qualidade.

\section{CONCLUSÕES}

- Todas as configurações de amostragem testadas, com áreas de unidades amostrais variando de 400 e $1.050 \mathrm{~m}^{2}$, resultaram em erros de amostragem relativos inferiores a $20 \%$, considerados satisfatórios para inventários de florestas nativas;

- Quanto maior a fração amostrada, mais precisas foram as estimativas para número de árvores e área basal;

- Para a estimativa do volume por hectare, a fração de amostragem a ser utilizada seria a menor, o que implicaria em um menor custo para a realização do inventário florestal;

- Os menores erros reais relativos para $N, G$ e $V$ foram encontrados para unidades amostrais com $1.000 \mathrm{~m}^{2}$;

- No entanto, cada variável estudada apresentou particularidades quanto aos três fatores analisados, principalmente quanto à forma;

- A forma das unidades amostrais influenciou os resultados, $N$ foi melhor estimado a partir de unidades amostrais mais largas, enquanto $G$ e $V$ a partir de unidades amostrais mais estreitas.

\section{REFERÊNCIAS}

AUGUSTYNCZIK, A. L. D. Avaliação do tamanho de parcelas e intensidades de amostragem para a estimativa de estoque e estrutura horizontal em um fragmento de Floresta Ombrófila Mista. 2011. 165 f. Dissertação (Mestrado) - Pós-Graduação em Engenharia Florestal, Universidade Federal do Paraná, Curitiba, 2011.

AUGUSTYNCZIK, A. L. D.; MACHADO, S. A.; FIGUEIREDO FILHO, A.; PÉLLICO NETTO, S. Avaliação do tamanho de parcelas e de intensidade de amostragem em inventários florestais. Scientia Forestalis, v. 41, n. 99, p. 361-368, 2013.
BONETES, L. Tamanho de parcelas e intensidade amostral para estimar o estoque e índices fitossociológicos em uma Floresta Ombrófila Mista. 2003. 111 f. Dissertação (Mestrado) - PósGraduação em Engenharia Florestal, Universidade Federal do Paraná, Curitiba, 2003.

CORTE, A. P. D.; SANQUETTA, C. R.; FIGUEIREDO FILHO, A.; PEREIRA, T. K.; BEHLING, A. Desempenho de métodos e processos de amostragem para avaliação de diversidade em Floresta Ombrófila Mista. Floresta, v. 43, n. 4, p. 579-582, 2013.

CORTE, A. P. D.; SANQUETTA, C.R.; RODRIGUES, A. L.; MOGNON. F. Dinâmica do crescimento, mortalidade e recrutamento na Floresta com Araucária. In: SANQUETTA, C. R.; CORTE, A. P. D.; RODRIGUES, A. L.; MOGNON, F. Floresta com Araucária: Pesquisas Ecológicas de Longa Duração. Curitiba: Multi-Graphic, 2014, 296 p.

DISPERATI, A. A. Mapeamento florestal da Floresta Nacional de Irati, PR. Curitiba: FUPEF, 1986. 18 p.

DURIGAN, G. Estrutura e diversidade de comunidades florestais. In: MARTINS, S. V. Ecologia de florestas tropicais do Brasil, Viçosa, 2009, p. 185-215.

FIGUEIREDO FILHO, A.; DIAS, A. N.; STEPKA, T. F.; SAWCZUK, A. R. Crescimento, mortalidade, ingresso e distribuição diamétrica em Floresta Ombrófila Mista. Floresta, v. 40, n. 4, p. 763-776, 2010.

HUSCH, B; MILLER, C. L.; BEERS, T. E. Forest mensuration. 3. ed. New York: J. Willey \& Sons, 1982. 397 p.

KERSTEN, R. A.; GALVÃO, F. Suficiência amostral em inventários florísticos e fitossociológicos. In: FELFILI, J. M.; EISENLOH, P. V.; MELO, M. M. R. F. Fitossociologia no Brasil: métodos e estudos de casos, Viçosa: UFV, 2011. v. 1, 558 p.

MAGURRAN, A. E. Medindo a diversidade biológica. Curitiba: Editora UFPR, 2011. $261 \mathrm{p}$.

MAZZA, C. A. S.; MAZZA, M. C. M.; SANTOS, J. E. SIG aplicado à caracterização ambiental de uma unidade de conservação: Floresta Nacional de Irati, Paraná. In: XII SIMPÓSIO BRASILEIRO DE SENSORIAMENTO REMOTO, 12., 2005, Goiânia. Anais... Goiânia: INPE, 2005. p. 2251-2258.

MORAIS FILHO, A. D.; BRAVO, C. V.; ROQUE, R. A. M.; ANDRADE, $W$. F. Utilização de métodos estatísticos em inventário florestal. ESALq-USP, Piracicaba, 2003. 27 p.

PÉLLICO NETTO, S.; BRENA, D. A. Inventário florestal. Curitiba: Editorado pelos autores, 1997. $316 \mathrm{p}$.

PÉLLICO NETTO, S.; ORELLANA, E.; LIMA, R.; FIGUEIREDO FILHO, A. Comportamento probabilístico dos raios das sextas árvores no método de Prodan e estimativas dos parâmetros dendrométricos para Araucaria angustifolia (Bertol.) Kuntze. Scientia Forestalis, v. 40, n. 96, p. 517-524, 2012.

PIZATTO, W. Avaliação biométrica da estrutura e da dinâmica de uma Floresta Ombrófila Mista em São João do Triunfo - PR; 1995 a 1998. 1999. 172 f. Dissertação (Mestrado) - Pós-Graduação em Engenharia Florestal, Universidade Federal do Paraná, Curitiba, 1999. 
ROCHA, M. B; SOUSA, T. B.; SILVA, J. G.; BARRETO, P. A. B. Comparação do censo com processos de amostragem para estimar o volume em povoamento de Pinus caribaea. In: IV CONEFLOR - III SEEFLOR, 2013, Vitória da Conquista. Anais... Vitória da Conquista: UESB, 2013, p. 306-309.

RODE, R. Avaliação florística e estrutural de uma Floresta Ombrófila Mista e de uma vegetação arbórea estabelecida sob um povoamento de Araucaria angustifolia de 60 anos. 2008. 122 f. Dissertação (Mestrado) - Pós-Graduação em Engenharia Florestal, Universidade Federal do Paraná, Curitiba, 2008.

ROIK, M. Dinâmica (2002-2011) e modelagem do incremento diamétrico em fragmento de Floresta Ombrófila Mista na Floresta Nacional de Irati, Paraná. 2012. 141 f. Dissertação (Mestrado) - Pós-Graduação em Engenharia Florestal, Universidade Estadual do Centro-Oeste, Irati, 2012.

SANQUETTA, C. R.; CORTE, A. P. D.; RODRIGUES, A. L.; WATZLAWICK, L. F. Inventários florestais: planejamento e execução. 4. ed. Curitiba: Multi-Graphic, 2014. 406 p.

SCOLFORO, J. R. S.; MELLO, J. M. Inventário florestal. Lavras: UFLA/Faepe, 2006. $561 \mathrm{p}$.

SINGH, K. D. Reconocimientos forestales: patrones de variación espacial en la selva tropical. Unasylva, v. 26, p. 18-23, 1974.

UBIALLI, J. A. Comparação de métodos e processos de amostragem para estudos fitossociológicos e estimativas de estoque de uma Floresta Ecotonal na região norte matogrossense. 2007. 241 f. Tese (Doutorado em Ciências Florestais) - Setor de Ciências Agrárias, Universidade Federal do Paraná, Curitiba, 2007.

UBIALLI, J. A.; FIGUEIREDO FILHO, A.; MACHADO, S. A.; ARCE, J. E. Comparação de métodos e processos de amostragem para estudos fitossociológicos em uma Floresta Ecotonal na região norte Matogrossense. Floresta, v. 39, p. 511-523, 2009.

VIBRANS, A. C; SEVEGNANI, L.; GASPER, A. L.; LIGNER, D. V. Inventário Florístico-Florestal de Santa Catarina. Volume III, Floresta Ombrófila Mista. Blumenau: Edifurb, 2013. 435 p. 